\title{
Soil chemical properties and growth performance of white seed melon (Cucumeropsis mannii Naudin) influenced by combined soil amendments in Ile-Oluji, Nigeria
}

\author{
S. L. Adebisi ${ }^{1 *}$, M. Yahqub ${ }^{2}$ and G. M. Akande ${ }^{3}$ \\ ${ }^{1}$ Department of Agricultural Technology, Federal Polytechnic, Ile-Oluji, Ondo State, Nigeria \\ ${ }^{2}$ Samaru College of Agriculture, Division of Agricultural Colleges, Ahmadu Bello University, Zaria, Nigeria \\ ${ }^{3}$ Department of Soil and Environment Management, Kogi State University, Ayingba, Kogi State, Nigeria \\ *Corresponding Author: sunadebisi@fedpolel.edu.ng / adebisisunday@gmail.com
}

\begin{abstract}
Field experiment was conducted to study the effects of crop residues in combination with NPK fertilizer (NPKF) on soil chemical properties and growth performance of white seed melon at Ile-Oluji, Ondo State Nigeria. The experiment involved applications of cocoa bean husk $(\mathrm{CBH})$, cocoa pod husk $(\mathrm{CPH})$, cocoa pod waste $(\mathrm{CPW})$, kola pod husk $(\mathrm{KPH})$, Tithonia diversifolia (weed much (WM) in combination with NPK 15:15:15 Fertilizer to produce twelve treatments at $4 \mathrm{t} \mathrm{ha}^{-1} \mathrm{CBH}+200 \mathrm{~kg} \mathrm{ha}{ }^{-1} \mathrm{NPKF}, 4$ $\mathrm{t} \mathrm{ha}^{-1} \mathrm{CBH}+100 \mathrm{~kg} \mathrm{ha}^{-1} \mathrm{NPKF}, 4 \mathrm{tha}^{-1} \mathrm{CPH}+200 \mathrm{~kg} \mathrm{ha}^{-1} \mathrm{NPKF}, 4 \mathrm{t} \mathrm{ha}^{-1} \mathrm{CPH}+100 \mathrm{~kg} \mathrm{ha}^{-1} \mathrm{NPKF}, 4 \mathrm{tha}{ }^{-1} \mathrm{CPW}+200 \mathrm{~kg} \mathrm{ha}{ }^{-1}$ NPKF, $4 \mathrm{t} \mathrm{ha}^{-1} \mathrm{CPW}+100 \mathrm{~kg} \mathrm{ha}^{-1} \mathrm{NPKF}, 4 \mathrm{t} \mathrm{ha}^{-1} \mathrm{KPH}+200 \mathrm{~kg} \mathrm{ha}^{-1} \mathrm{NPKF}, 4 \mathrm{t} \mathrm{ha}^{-1} \mathrm{KPH}+100 \mathrm{~kg} \mathrm{ha}^{-1} \mathrm{NPKF}, 2 \mathrm{tha}{ }^{-1} \mathrm{WM}+200$ $\mathrm{kg} \mathrm{ha}^{-1} \mathrm{NPKF}, 2 \mathrm{t} \mathrm{ha}^{-1} \mathrm{WM}+100 \mathrm{~kg} \mathrm{ha}^{-1} \mathrm{NPKF}, 300 \mathrm{~kg} \mathrm{ha}^{-1} \mathrm{NPKF}$ and control, all the amendments tested significantly improved soil chemical properties and growth performance of white seed melon relative to control. Three cocoa plants residues in combination with NPKF at reduced rates $4 \mathrm{t}$ ha $\mathrm{CBH}+200 \mathrm{~kg} \mathrm{ha}^{-1} \mathrm{NPKF}, 4 \mathrm{tha}^{-1} \mathrm{CPW}+200 \mathrm{~kg} \mathrm{ha}^{-1} \mathrm{NPKF}$ and $4 \mathrm{t} \mathrm{ha}{ }^{-1} \mathrm{CPH}+200 \mathrm{~kg} \mathrm{ha}{ }^{-1}$ $\mathrm{NPKF}$ respectively significantly $(\mathrm{p}<0.05$ ) improved soil $\mathrm{pH}$, organic matter $(\mathrm{OM})$, available $\mathrm{P}$, exchangeable $\mathrm{k}, \mathrm{Ca}$ and $\mathrm{Mg}, \mathrm{Na}, \mathrm{Fe}$, $\mathrm{Al}$ and ECEC among the amendments tested. All treatments increased number of leaf, branches and vine length of white seed melon compared to control. $4 \mathrm{t} \mathrm{ha}^{-1} \mathrm{CBH}+200 \mathrm{~kg} \mathrm{ha}^{-1} \mathrm{NPKF}, 4 \mathrm{t} \mathrm{ha}^{-1} \mathrm{CPW}+200 \mathrm{~kg} \mathrm{ha}^{-1} \mathrm{NPKF}, 4 \mathrm{t} \mathrm{ha}{ }^{-1} \mathrm{CPH}+200 \mathrm{~kg} \mathrm{ha}^{-1} \mathrm{NPKF}$, had highest value of crop branches.
\end{abstract}

Keywords: White seed melon, Crop residue, Combined amendments, Soil chemical properties, Growth performance.

\section{INTRODUCTION}

Soil fertility management is one of the major constraints affecting the quality and quantity of agricultural production in developing countries, particularly in Nigeria. Crop quality and quantity are largely dependent on the ability of soil to supply essential nutrient elements for plant growth without a toxic concentration (Boyd, 1996). Soils of the tropics varied widely in physical and chemical properties. In West Africa soils of rainforest are generally acidic and of low to medium inherent fertility. Crop varieties with the most high yielding potential cultivated on such soils without regular replenishment of nutrients either through organic or inorganic means are bound to experience low crop quality, yield or total failure due to nutrient loss through crop removal, leaching and erosion. The use of chemical fertilizers to return lost nutrients and achieve high crop yield is unsustainable due to its scarcity and high cost (Ojeniyi, 2000). Although inorganic fertilizers ensure quick availability of nutrients to crops but they have limited residual effect of the applied nutrients (Okigbo, 2000). In addition, inorganic fertilizer has not been helpful in intensive agriculture because it is often associated with a reduction in crop yield, soil acidity, nutrient imbalance and degradation of soil physical attributes. Other limitations include nutrients leaching, erosion and volatilization (Ojeniyi, 2000). Chemical fertilizers are said to have the following characteristics (Ellison, 2011); Water solubility of chemical fertilizers results in fast nutrient release. Death of Nitrogen fixing bacterium, the organism that plays a central role in refurbishing a maturing plants nitrogen demands is part of the results of chemical fertilizers with high acid content like sulphuric and hydrochloric acids which when successfully going through soil acidity. Nutrient supply through the application of organic fertilizers is also faced with some challenges. The maintenance of organic matter in soil is constraint by the high competing demands of organic and agricultural wastes. Organic fertilizers are known to be slow released nutrient sources. This clearly shows that nutrient immobilization may cause crops to experience initial nutrient deprivation before mineralization. They are also required in large quantities which may not be readily available to farmers (Agbede \& Kalu, 1995; Okigbo, 2000; Adekiya et al., 2012). Combination of crop or plant residue with synthetic fertilizers is the sustainable means of avoiding problems emanating from sole use of organic and inorganic fertilizers. Organic fertilizer was reported to improve soil characteristics and obtain high crop yields in addition to inorganic fertilizer (Cezar, 2004). Akanbi et al. 
(2013) and Ojeniyi \& Adeniyan (1999) recognized the need to intensify studies into locally sourced, cheap, adoptable organic sources of plant nutrients. Babadele \& Ojeniyi (2013) found that plant variety such as those of siam weed and sawdust used alone or combined further with NPK fertilizer at a reduced rate supplied nutrients and improved yield. Organic amendments discharge their nourishing contents only when they break down through the intricate ecology of living creatures in the soil at that time they steadily discharge contents. All the components in the organic amendments are completely essential soil nutrients. The fact that the material is organic signifies that it is derived from a once-living plant, animal or a mix of both, which assures us that all components there are crucial to life. The organic amendment is relatively cheaper and it has all the carbon and vitality to conform to the demands of soil microbes. The above characteristic of organic and inorganic fertilizers which complements each other makes its combination a necessary component for crop production. While the importance of the mixture has been demonstrated (Onunka \& Nwokocha, 2003). Mann's cucumeropsis (Cucumeropsis mannii Naudin) is one of the most important species of melon. It is an important quality crop. The large white seeds produced by the crop is called egusi-itoo. The seeds are processed into soups and oil products and are also eaten individually as a snack (National Research Council, 2006). The benefits of white seed melon are many but soil fertility is one of the most important factors limiting its production. It is a very high nutrient - demanding crop and it requires adequate nutrition for maximum performance (National Research Council, 2006). A complete fertilizer should be applied before the propagation of white seed melon with periodical application of nitrogenous fertilizer (National Research Council, 2006). The growing of Cucumeropsis mannii in savannahs with low fertility and organic matter was reported to be more challenging (National Research Council, 2006). The objective of this experiment was to determine the effect of combined amendments on soil properties and growth performance of white seed melon.

\section{MATERIALS AND METHODS}

The study was conducted during 2018 cropping season at the Teaching and Research Farm, Federal Polytechnic Ile Oluji, Ondo State located in the rainforest Zone of Nigeria on latitude $7^{\circ} 20^{\prime} \mathrm{N}$ and longitude $4^{\circ} 87^{\prime} \mathrm{E}$ and Altitude of 247 metres. The location has a bimodal rainfall of 1250 to $1460 \mathrm{~mm}$ with mean annual rainfall of $1367 \mathrm{~mm}$ and average number of rainy days of about 112 per annum. Temperature is almost uniform throughout the year $23-32{ }^{\circ} \mathrm{C}$ with little deviation from mean annual of $27^{\circ} \mathrm{C}$. February and March are the hottest months with mean temperature of $28^{\circ} \mathrm{C}$ and $27^{\circ} \mathrm{C}$, respectively. The mean annual radiation is about $130 \mathrm{kcal} \mathrm{cm}^{-3}$ with total sunshine hour is about 2000 hours year

1. The location is found within the high forest zone where the rich tropical forests once thrived. The location has a tropical humid climate with distinct wet and dry seasons. Its wet season starts from late March to October while little dry season occurs in August.

\section{Experimental design and treatments}

The experiment was arranged in a randomized complete block with each treatment replicated four times. The area used was $24 \mathrm{~m} \times 49 \mathrm{~m}\left(1176 \mathrm{~m}^{2}\right)$ in total. Each plot measured $4 \mathrm{~m} \times 3 \mathrm{~m}$ with discard of $1 \mathrm{~m}$ within the plots and $2 \mathrm{~m}$ between the block. The trial involved application of $4 \mathrm{t} \mathrm{ha}^{-1}$ cocoa bean husk $(\mathrm{CBH})$; cocoa pod husk (CPH); cocoa pod waste (CPW); Kola pod husk (KPH) and $2 \mathrm{t} \mathrm{ha}^{-1}$ Tithonia diversifolia (weed mulch (WM) plus 200 or $100 \mathrm{~kg} \mathrm{ha}^{-1} \mathrm{NPK}$ 15:15:15 fertilizer (NPKF) and $300 \mathrm{~kg} \mathrm{ha}^{-1} \mathrm{NPKF}$ and control (no amendment). Two seeds of white seeds melon were planted per hole and later thinned to one at a spacing of $1 \mathrm{~m} \times 1 \mathrm{~m}$ with a total of twenty (20) plants per plot. Before the commencement of the experiment, soil samples randomly collected from $0-20 \mathrm{~cm}$ depth were thoroughly mixed to form a composite which was later analyzed for physical and chemical properties. At the harvest, another set of composite samples were collected per plot basis and similarly analyzed for routine chemical analysis as described by Carter (1993). The soil samples were air-dried and sieved using a $2 \mathrm{~mm}$ sieve before making the determinations. Soil organic matter was determined by the procedure of Walkley and Black using the dichromate wet oxidation method (Nelson \& Sommers, 1996), total $\mathrm{N}$ was determined by micro-Kjeldahl digestion method (Bremner, 1996), available $\mathrm{P}$ was determined by Bray-1 extraction followed by molybdenum blue colorimetry (Frank et al., 1998). Exchangeable K, Ca and $\mathrm{Mg}$ were extracted using $1.0 \mathrm{~N}$ ammonium acetate. Thereafter, $\mathrm{K}$ was determined using a flame photometer and $\mathrm{Ca}$ and $\mathrm{Mg}$ were determined by EDTA titration method (Hendershot \& Lalande, 1993). The digital electronic $\mathrm{pH}$ meter was used to determine soil $\mathrm{pH}$ in soil water (1:2) medium. Bouyoucos hydrometer method was used for particle size analysis (Sheldrick \& Wang, 1993).

\section{Data collection and statistical analysis}

Five plants of white seed melon were randomly selected from each plot across the four blocks for growth determination. The parameters assessed included the number of leaves, number of branches and vine length. The data collected were subjected to analysis of variance (ANOVA) using the SPSS package (version 16) and treatment means were compared using the Duncan's multiple range test (DMRT). 


\section{RESULTS}

\section{Pre-Planting Soil properties}

Soil properties of the experimental site before planting is presented in table 1 . The result revealed that the soil at the location was sandy loam, acidic, low in $\mathrm{N}$, available $\mathrm{P}$, exchangeable $\mathrm{K}$ and $\mathrm{OM}$ in accordance with the rating of Akinrinde \& Obigbesan (2000). Therefore, the results revealed that additional soil conditioner would be needed before the soil could effectively produce a crop.

Table 1. Pre-Planting Soil properties.

\begin{tabular}{ll}
\hline Property & Values \\
\hline Sand $(\%)$ & 73.9 \\
Silt $(\%)$ & 14.7 \\
Clay $(\%)$ & 11.4 \\
Textural Class & Sandy loam \\
pH $\left(\mathrm{H}_{2} 0\right)$ & 5.83 \\
Organic Matter $(\%)$ & 2.36 \\
Total N $\left(\mathrm{gkg}^{-1}\right)$ & 0.20 \\
Available P $\left(\mathrm{mgkg}^{-1}\right)$ & 15.14 \\
Exchangeable K $\left(\mathrm{cmolkg}^{-1}\right)$ & 0.17 \\
Exchangeable Ca $\left(\mathrm{cmolkg}^{-1}\right)$ & 2.61 \\
Exchangeable Mg $\left(\mathrm{cmolkg}^{-1}\right)$ & 1.24 \\
Na $(\mathrm{cmolkg})$ & 0.58 \\
$\mathrm{H}(\mathrm{cmolkg})$ & 1.29 \\
$\mathrm{Al}(\mathrm{cmolkg}$ & -1 \\
ECEC & 0.81 \\
BS $(\%)$ & 6.7 \\
\hline Note: BS = Base saturation. & 59.31 \\
\hline
\end{tabular}

Data on effects of combined application of amendments on number of leaf of white seed melon are shown in table 2. Number of leaf at 4, 12, 20, and 28 weeks after treatment application (WATA) ranged from (7.2-13.1), (47.67-68.11), (133.27-174.71) and (210.47-274.57) respectively. All the amendments tested significantly improved the number of leaf of white seed melon relative to the control. The highest number of leaf (13.13) was obtained for plant treated with $4 \mathrm{t}$ $\mathrm{ha}^{-1} \mathrm{CBH}+200 \mathrm{~kg} \mathrm{ha}^{-1} \mathrm{NPKF}$ and it was closely followed by the plant treated with $4 \mathrm{t} \mathrm{ha}^{-1} \mathrm{CPW}+200 \mathrm{~kg} \mathrm{ha}^{-1} \mathrm{NPKF}$. Plants treated with $4 \mathrm{t} \mathrm{ha}^{-1} \mathrm{CBH}+200 \mathrm{~kg} \mathrm{ha}^{-1} \mathrm{NPKF}, 4 \mathrm{t} \mathrm{ha}^{-1} \mathrm{CPW}+200 \mathrm{~kg} \mathrm{ha}^{-1} \mathrm{NPKF}, 4 \mathrm{t} \mathrm{ha}^{-1} \mathrm{CPW}+100 \mathrm{~kg} \mathrm{ha}{ }^{-1}$ $\mathrm{NPKF}$, and $4 \mathrm{t} \mathrm{ha}^{-1} \mathrm{KPH}+200 \mathrm{~kg} \mathrm{ha}^{-1} \mathrm{NPKF}$ were not significantly different from one another at $(\mathrm{P}<0.05)$ at 4 WATA. Highest values of number of leaf $\left(68.11,174.77\right.$ and 274.57) were obtained for $300 \mathrm{~kg} \mathrm{ha}^{-1} \mathrm{NPKF}$ at 12 and 20 WATA and $4 \mathrm{t} \mathrm{ha}^{-1} \mathrm{CPW}+200 \mathrm{~kg} \mathrm{ha}^{-1} \mathrm{NPKF}$ at $28 \mathrm{WATA}$. The number of leaf recorded for $300 \mathrm{~kg} \mathrm{ha}^{-1} \mathrm{NPKF}$ was not significantly different from the plant treated with $4 \mathrm{t} \mathrm{ha}^{-1} \mathrm{CBH}+200 \mathrm{~kg} \mathrm{ha}^{-1} \mathrm{NPKF}$ and $4 \mathrm{t} \mathrm{ha}^{-1} \mathrm{CPW}+200 \mathrm{~kg} \mathrm{ha}{ }^{-1}$ NPKF at 12 WATA. The number of leaf at 20 WATA showed those plant residues in combination with either 100 or $200 \mathrm{~kg} \mathrm{ha}^{-1} \mathrm{NPKF}$ were not significantly higher than one another. Plot treated with $2 \mathrm{t} \mathrm{ha}^{-1} \mathrm{WM}+100 \mathrm{~kg} \mathrm{ha}{ }^{-1} \mathrm{NPKF}$ was significantly higher than the number of leaf obtained for the control. The value obtained on the number of leaf at 28 WATA revealed that plant treated with $4 \mathrm{t} \mathrm{ha}^{-1} \mathrm{CPW}+200 \mathrm{~kg} \mathrm{ha}^{-1} \mathrm{NPKF}$ and $300 \mathrm{~kg} \mathrm{ha}^{-1} \mathrm{NPKF}$ were statistically similar and showed no significant difference from each other at $(\mathrm{P}<0.05)$.

Table 2. Effects of combined amendments on number of leaf of white seed melon (Cucumeropsis mannii Naudin) at 4, 12, 20 and 28 WATA at Ile-Oluji.

\begin{tabular}{lllll}
\hline Treatments & \multicolumn{4}{c}{ Weeks After Treatment Application (WATA) } \\
\cline { 2 - 5 } & $\mathbf{4}$ & $\mathbf{1 2}$ & $\mathbf{2 0}$ & $\mathbf{2 8}$ \\
\hline Control & $7.2 \mathrm{e}$ & $47.67 \mathrm{e}$ & $133.27 \mathrm{c}$ & $210.47 \mathrm{~h}$ \\
$300 \mathrm{~kg} \mathrm{ha}^{-1} \mathrm{NPKF}$ & $12.90 \mathrm{ab}$ & $68.10 \mathrm{a}$ & $174.77 \mathrm{a}$ & $274.0 \mathrm{ab}$ \\
$4 \mathrm{t} \mathrm{ha}^{-1} \mathrm{CBH}+200 \mathrm{~kg} \mathrm{ha}^{-1} \mathrm{NPKF}$ & $13.13 \mathrm{ab}$ & $63.71 \mathrm{abc}$ & $174.2 \mathrm{a}$ & $272.33 \mathrm{abcd}$ \\
$4 \mathrm{t} \mathrm{ha}^{-1} \mathrm{CBH}+100 \mathrm{~kg} \mathrm{ha}^{-1} \mathrm{NPKF}$ & $11.87 \mathrm{~cd}$ & $67.42 \mathrm{~cd}$ & $164.67 \mathrm{ab}$ & $267.8 \mathrm{cde}$ \\
$4 \mathrm{t} \mathrm{ha}^{-1} \mathrm{CPH}+200 \mathrm{~kg} \mathrm{ha}^{-1} \mathrm{NPKF}$ & $12.03 \mathrm{~cd}$ & $65.02 \mathrm{bcd}$ & $167.2 \mathrm{ab}$ & $268.17 \mathrm{cde}$ \\
$4 \mathrm{t} \mathrm{ha}^{-1} \mathrm{CPH}+100 \mathrm{~kg} \mathrm{ha}^{-1} \mathrm{NPKF}$ & $11.44 \mathrm{~d}$ & $62.13 \mathrm{~d}$ & $160.47 \mathrm{ab}$ & $264.37 \mathrm{efg}$ \\
$4 \mathrm{t} \mathrm{ha}^{-1} \mathrm{CPW}+200 \mathrm{~kg} \mathrm{ha}^{-1} \mathrm{NPKF}$ & $13.07 \mathrm{ab}$ & $67.34 \mathrm{abcd}$ & $171.2 \mathrm{a}$ & $274.57 \mathrm{a}$ \\
$4 \mathrm{t} \mathrm{ha}^{-1} \mathrm{CPW}+100 \mathrm{~kg} \mathrm{ha}^{-1} \mathrm{NPKF}$ & $12.53 \mathrm{abc}$ & $64.12 \mathrm{bcd}$ & $166.8 \mathrm{ab}$ & $267.64 \mathrm{def}$ \\
$4 \mathrm{t} \mathrm{ha}^{-1} \mathrm{KPH}+200 \mathrm{~kg} \mathrm{ha}^{-1} \mathrm{NPKF}$ & $12.47 \mathrm{abc}$ & $61.93 \mathrm{bcd}$ & $166.37 \mathrm{ab}$ & $268.83 \mathrm{cde}$ \\
$4 \mathrm{t} \mathrm{ha}^{-1} \mathrm{KPH}+100 \mathrm{~kg} \mathrm{ha}^{-1} \mathrm{NPKF}$ & $12.03 \mathrm{~cd}$ & $58.49 \mathrm{~d}$ & $161.6 \mathrm{ab}$ & $262.7 \mathrm{fg}$ \\
$2 \mathrm{t} \mathrm{ha}^{-1} \mathrm{WM}+200 \mathrm{~kg} \mathrm{ha}^{-1} \mathrm{NPKF}$ & $11.97 \mathrm{~cd}$ & $61.84 \mathrm{~d}$ & $166.43 \mathrm{ab}$ & $267.47 \mathrm{def}$ \\
$2 \mathrm{t} \mathrm{ha}^{-1} \mathrm{WM}+100 \mathrm{~kg} \mathrm{ha}^{-1} \mathrm{NPKF}$ & $11.3 \mathrm{~d}$ & $58.03 \mathrm{~d}$ & $141.21 \mathrm{~b}$ & $261.37 \mathrm{~g}$ \\
\hline
\end{tabular}

Note: NPKF= NPK 15:15:15 fertilizer; CBH= Cocoa Bean Husk; CPH= Cocoa Pod Husk; CPW= Cocoa Pod Waste; KPH= Kola Pod Husk; WM= Weed Mulch. Means with the same letters are not significantly different $(P<0.05)$ from other. 
The result on the effect of soil amendment on number of branches of white seed melon is shown in table 3 . The number of branches ranged from (1.57-1.83), (3.07-5.50), (5.67-10.33) and (10.04-12.07) at 4, 12, 20 and 28 WATA respectively. Highest value of number of branches of white seed melon was obtained for plant treated with $300 \mathrm{~kg}^{-1}$ NPKF, $4 \mathrm{t} \mathrm{ha}^{-1} \mathrm{CPW}+200 \mathrm{~kg} \mathrm{ha}^{-1} \mathrm{NPKF}, 4 \mathrm{t} \mathrm{ha}^{-1} \mathrm{CPW}+200 \mathrm{~kg} \mathrm{ha}^{-1} \mathrm{NPKF}$ and $4 \mathrm{t} \mathrm{ha}^{-1} \mathrm{CBH}+200 \mathrm{~kg} \mathrm{ha}{ }^{-1} \mathrm{NPKF}$ and $4 \mathrm{t} \mathrm{ha}^{-1} \mathrm{CBH}+200 \mathrm{~kg} \mathrm{ha}^{-1} \mathrm{NPKF}, 4 \mathrm{t} \mathrm{ha}^{-1} \mathrm{CPH}+200 \mathrm{~kg} \mathrm{ha}^{-1} \mathrm{NPKF}$ and $4 \mathrm{t} \mathrm{ha}^{-1} \mathrm{CPW}+200 \mathrm{~kg} \mathrm{ha}^{-1} \mathrm{NPKF}$ at $4,12,20$ and 28 WATA respectively. Value on the number of branches of white seed melon recorded for $300 \mathrm{~kg} \mathrm{ha}^{-1} \mathrm{NPKF}$ was not significantly different from plant treated with other soil amendments at 4 and 12 WATA. Significant difference was only observed at 20 and 28 WATA compared with $300 \mathrm{~kg} \mathrm{ha}^{-1} \mathrm{NPKF}, 4 \mathrm{t} \mathrm{ha}^{-1} \mathrm{KPH}$ and $2 \mathrm{t} \mathrm{ha}^{-1} \mathrm{WM}$ plus 100 or $200 \mathrm{~kg}$ $\mathrm{ha}^{-1}$ NPKF and that of control.

Table 3. Effects of combined amendments on number of branches of white seed melon (Cucumeropsis mannii Naudin) at 4, 12,20 and 28 WATA at Ile-Oluji.

\begin{tabular}{lllll}
\hline Treatments & \multicolumn{3}{c}{ Weeks After Treatment Application (WATA) } \\
\cline { 2 - 5 } & $\mathbf{4}$ & $\mathbf{1 2}$ & $\mathbf{2 0}$ & $\mathbf{2 8}$ \\
\hline Control & $1.57 \mathrm{e}$ & $3.07 \mathrm{~b}$ & $5.67 \mathrm{e}$ & $10.04 \mathrm{c}$ \\
$300 \mathrm{~kg} \mathrm{ha}^{-1} \mathrm{NPKF}$ & $1.93 \mathrm{a}$ & $5.47 \mathrm{a}$ & $10.27 \mathrm{a}$ & $11.87 \mathrm{a}$ \\
$4 \mathrm{t} \mathrm{ha}^{-1} \mathrm{CBH}+200 \mathrm{~kg} \mathrm{ha}^{-1} \mathrm{NPKF}$ & $1.83 \mathrm{abc}$ & $5.40 \mathrm{a}$ & $10.33 \mathrm{a}$ & $12.00 \mathrm{a}$ \\
$4 \mathrm{t} \mathrm{ha}^{-1} \mathrm{CBH}+100 \mathrm{~kg} \mathrm{ha}^{-1} \mathrm{NPKF}$ & $1.80 \mathrm{abc}$ & $5.40 \mathrm{a}$ & $10.27 \mathrm{a}$ & $11.93 \mathrm{a}$ \\
$4 \mathrm{t} \mathrm{ha}^{-1} \mathrm{CPH}+200 \mathrm{~kg} \mathrm{ha}^{-1} \mathrm{NPKF}$ & $1.82 \mathrm{abc}$ & $5.43 \mathrm{a}$ & $10.20 \mathrm{a}$ & $12.07 \mathrm{a}$ \\
$4 \mathrm{t} \mathrm{ha}^{-1} \mathrm{CPH}+100 \mathrm{~kg} \mathrm{ha}^{-1} \mathrm{NPKF}$ & $1.80 \mathrm{abc}$ & $5.43 \mathrm{a}$ & $10.20 \mathrm{a}$ & $12.03 \mathrm{a}$ \\
$4 \mathrm{t} \mathrm{ha}^{-1} \mathrm{CPW}+200 \mathrm{~kg} \mathrm{ha}^{-1} \mathrm{NPKF}$ & $1.82 \mathrm{abc}$ & $5.50 \mathrm{a}$ & $10.33 \mathrm{a}$ & $12.07 \mathrm{a}$ \\
$4 \mathrm{t} \mathrm{ha}^{-1} \mathrm{CPW}+100 \mathrm{~kg} \mathrm{ha}^{-1} \mathrm{NPKF}$ & $1.80 \mathrm{abc}$ & $5.47 \mathrm{a}$ & $10.27 \mathrm{a}$ & $11.93 \mathrm{a}$ \\
$4 \mathrm{t} \mathrm{ha}^{-1} \mathrm{KPH}+200 \mathrm{~kg} \mathrm{ha}^{-1} \mathrm{NPKF}$ & $1.81 \mathrm{abc}$ & $5.13 \mathrm{a}$ & $8.63 \mathrm{c}$ & $11.00 \mathrm{~b}$ \\
$4 \mathrm{t} \mathrm{ha}^{-1} \mathrm{KPH}+100 \mathrm{~kg} \mathrm{ha}^{-1} \mathrm{NPKF}$ & $1.80 \mathrm{abc}$ & $5.17 \mathrm{a}$ & $8.23 \mathrm{~d}$ & $11.03 \mathrm{~b}$ \\
$2 \mathrm{t} \mathrm{ha}^{-1} \mathrm{WM}+200 \mathrm{~kg} \mathrm{ha}^{-1} \mathrm{NPKF}$ & $1.80 \mathrm{abc}$ & $4.93 \mathrm{a}$ & $8.43 \mathrm{~d}$ & $11.00 \mathrm{~b}$ \\
$2 \mathrm{t} \mathrm{ha}^{-1} \mathrm{WM}+100 \mathrm{~kg} \mathrm{ha}^{-1} \mathrm{NPKF}$ & $1.63 \mathrm{de}$ & $4.87 \mathrm{a}$ & $7.73 \mathrm{~d}$ & $10.87 \mathrm{~b}$ \\
\hline
\end{tabular}

Note: $\mathrm{NPKF}=\mathrm{NPK}$ 15:15:15 fertilizer; $\mathrm{CBH}=$ Cocoa Bean Husk; CPH= Cocoa Pod Husk; CPW= Cocoa Pod Waste;

$\mathrm{KPH}=$ Kola Pod Husk; WM= Weed Mulch. Means with the same letters are not significantly different $(P<0.05)$ from other.

The results on effects of amendments on vine length of white seed melon are shown in table 4. All the amendments tested significantly improved the vine length of white seed melon relative to the control. Vine length of melon ranged from (14.96-19.40), (15.67-253.00), (228.83-623.87) and (287.20-844.3) at 4, 12, 20 and 28 WATA respectively. Value of vine length of white seed melon recorded revealed that all soil amendments were not significantly different $(\mathrm{P}<$ 0.05 ) from one another but significantly higher than the control at 4 WATA. The highest value of vine length was obtained for plant amended with $4 \mathrm{t} \mathrm{ha}^{-1} \mathrm{NPKF}$ at 12 WATA and $300 \mathrm{~kg} \mathrm{ha}^{-1}$ NPKF at 20 and 28 WATA but there was no significant difference compared with 4 t/ha CBH, CPH and CPW plus 200 or $100 \mathrm{~kg} \mathrm{ha}^{-1} \mathrm{NPKF}$.

Table 4. Effects of combined amendments on vine length of white seed melon (Cucumeropsis mannii Naudin) at 4, 12, 20 and 28 WATA at Ile-Oluji.

\begin{tabular}{lllll}
\hline Treatments & \multicolumn{4}{c}{ Weeks After Treatment Application (WATA) } \\
\cline { 2 - 5 } & $\mathbf{4}$ & $\mathbf{1 2}$ & $\mathbf{2 0}$ & $\mathbf{2 8}$ \\
\hline Control & $14.96 \mathrm{~b}$ & $156.67 \mathrm{f}$ & $228.83 \mathrm{f}$ & $287.20 \mathrm{~d}$ \\
$300 \mathrm{~kg} \mathrm{ha}^{-1} \mathrm{NPKF}$ & $19.40 \mathrm{a}$ & $252.60 \mathrm{a}$ & $623.87 \mathrm{ab}$ & $844.30 \mathrm{a}$ \\
$4 \mathrm{t} \mathrm{ha}^{-1} \mathrm{CBH}+200 \mathrm{~kg} \mathrm{ha}^{-1} \mathrm{NPKF}$ & $19.33 \mathrm{a}$ & $244.30 \mathrm{ab}$ & $605.20 \mathrm{ab}$ & $814.23 \mathrm{abc}$ \\
$4 \mathrm{t} \mathrm{ha}^{-1} \mathrm{CBH}+100 \mathrm{~kg} \mathrm{ha}^{-1} \mathrm{NPKF}$ & $18.96 \mathrm{a}$ & $233.63 \mathrm{abc}$ & $575.17 \mathrm{abcd}$ & $793.37 \mathrm{abc}$ \\
$4 \mathrm{t} \mathrm{ha}^{-1} \mathrm{CPH}+200 \mathrm{~kg} \mathrm{ha}^{-1} \mathrm{NPKF}$ & $19.00 \mathrm{a}$ & $239.70 \mathrm{abc}$ & $599.07 \mathrm{ab}$ & $812.33 \mathrm{abc}$ \\
$4 \mathrm{t} \mathrm{ha}^{-1} \mathrm{CPH}+100 \mathrm{~kg} \mathrm{ha}^{-1} \mathrm{NPKF}$ & $18.73 \mathrm{a}$ & $227.07 \mathrm{abcd}$ & $5.7853 \mathrm{abcd}$ & $791.83 \mathrm{abc}$ \\
$4 \mathrm{t} \mathrm{ha}^{-1} \mathrm{CPW}+200 \mathrm{~kg} \mathrm{ha}^{-1} \mathrm{NPKF}$ & $19.33 \mathrm{a}$ & $253.00 \mathrm{a}$ & $620.00 \mathrm{ab}$ & $8.4173 \mathrm{a}$ \\
$4 \mathrm{t} \mathrm{ha}^{-1} \mathrm{CPW}+100 \mathrm{~kg} \mathrm{ha}^{-1} \mathrm{NPKF}$ & $19.20 \mathrm{a}$ & $237.97 \mathrm{abc}$ & $613.67 \mathrm{ab}$ & $819.33 \mathrm{abc}$ \\
$4 \mathrm{t} \mathrm{ha}^{-1} \mathrm{KPH}+200 \mathrm{~kg} \mathrm{ha}^{-1} \mathrm{NPKF}$ & $18.70 \mathrm{a}$ & $214.43 \mathrm{cde}$ & $5.7140 \mathrm{bcde}$ & $806.73 \mathrm{abc}$ \\
$4 \mathrm{t} \mathrm{ha}^{-1} \mathrm{KPH}+100 \mathrm{~kg} \mathrm{ha}^{-1} \mathrm{NPKF}$ & $18.50 \mathrm{a}$ & $206.63 \mathrm{de}$ & $5.4943 \mathrm{cde}$ & $796.77 \mathrm{abc}$ \\
$2 \mathrm{t} \mathrm{ha}^{-1} \mathrm{WM}+200 \mathrm{~kg} \mathrm{ha}^{-1} \mathrm{NPKF}$ & $17.56 \mathrm{a}$ & $205.10 \mathrm{de}$ & $546.63 \mathrm{cde}$ & $779.80 \mathrm{bc}$ \\
$2 \mathrm{t} \mathrm{ha}^{-1} \mathrm{WM}+100 \mathrm{~kg} \mathrm{ha}^{-1} \mathrm{NPKF}$ & $18.73 \mathrm{a}$ & $199.30 \mathrm{e}$ & $526.50 \mathrm{e}$ & $768.10 \mathrm{c}$ \\
\hline
\end{tabular}

Note: $\mathrm{NPKF}=$ NPK 15:15:15 fertilizer; $\mathrm{CBH}=$ Cocoa Bean Husk; CPH= Cocoa Pod Husk; CPW= Cocoa Pod Waste; $\mathrm{KPH}=$ Kola Pod Husk; WM= Weed Mulch. Means with the same letters are not significantly different $(P<0.05)$ from other.

Data on soil chemical properties after harvest of white seed melon are shown in tables 5, 6 and 7. All soil amendments imposed significantly improved soil chemical properties relative to the control. Data obtained on soil pH $\left(\mathrm{H}_{2} 0\right), \mathrm{OM}, \mathrm{N}, \mathrm{P}, \mathrm{K}, \mathrm{Ca}, \mathrm{Mg}, \mathrm{Na}, \mathrm{H}^{+}, \mathrm{Al}, \mathrm{ECEC}$ and $\mathrm{BS}$ ranged from, (5.06-6.57), (1.85-4.76\%), (0.20-0.93\%), (7.8825.92\%), (0.41-0.91 $\left.\mathrm{cmol} \mathrm{kg}^{-1}\right),\left(1.42-1.73 \mathrm{cmol} \mathrm{kg}{ }^{-1}\right),\left(0.37-0.79 \mathrm{cmol} \mathrm{kg}{ }^{-1}\right),\left(0.69-0.75 \mathrm{cmol} \mathrm{kg}^{-1}\right),\left(1.51-1.97 \mathrm{cmol} \mathrm{kg}^{-}\right.$ 
$\left.{ }^{1}\right),\left(0.41-0.75 \mathrm{cmol} \mathrm{kg}^{-1}\right),\left(5.356 .29 \mathrm{cmol} \mathrm{kg}^{-1}\right)$ and $(50.44-64.92 \%)$. Highest value of soil $\mathrm{pH}\left(\mathrm{H}_{2} 0\right)$ was obtained for the plot treated with $4 \mathrm{tha}^{-1} \mathrm{CPH}+200 \mathrm{~kg} \mathrm{ha}^{-1} \mathrm{NPKF}$ and it was significantly higher than the $\mathrm{pH}$ value recorded for other soil amendments. Least value of soil $\mathrm{pH}$ was recorded for the control and it was significantly lower than all the soil amendments imposed tables 5 and 6.

Table 5. Effects of combined amendments on soil $\mathrm{pH}, \mathrm{OM}, \mathrm{N}$ and $\mathrm{P}$ at Ile-Oluji.

\begin{tabular}{lllll}
\hline Treatments & $\mathbf{p H}\left(\mathbf{H}_{\mathbf{2}} \mathbf{0}\right)$ & $\mathbf{O M}(\boldsymbol{\%})$ & $\mathbf{N}(\boldsymbol{\%})$ & $\mathbf{P}(\boldsymbol{\%})$ \\
\hline Control & $5.06 \mathrm{k}$ & $1.85 \mathrm{j}$ & 0.201 & $7.88 \mathrm{k}$ \\
$300 \mathrm{~kg} \mathrm{ha}^{-1} \mathrm{NPKF}$ & $5.41 \mathrm{o}$ & $2.14 \mathrm{i}$ & $0.89 \mathrm{c}$ & $25.92 \mathrm{a}$ \\
$4 \mathrm{t} \mathrm{ha}^{-1} \mathrm{CBH}+200 \mathrm{~kg} \mathrm{ha}^{-1} \mathrm{NPKF}$ & $6.52 \mathrm{c}$ & $4.76 \mathrm{a}$ & $0.90 \mathrm{~b}$ & $20.07 \mathrm{~h}$ \\
$4 \mathrm{t} \mathrm{ha}^{-1} \mathrm{CBH}+100 \mathrm{~kg} \mathrm{ha}^{-1} \mathrm{NPKF}$ & $6.32 \mathrm{f}$ & $4.76 \mathrm{a}$ & $0.90 \mathrm{~b}$ & $23.69 \mathrm{c}$ \\
$4 \mathrm{t} \mathrm{ha}^{-1} \mathrm{CPH}+200 \mathrm{~kg} \mathrm{ha}^{-1} \mathrm{NPKF}$ & $6.57 \mathrm{a}$ & $4.74 \mathrm{~b}$ & $0.93 \mathrm{a}$ & $24.31 \mathrm{~b}$ \\
$4 \mathrm{t} \mathrm{ha}^{-1} \mathrm{CPH}+100 \mathrm{~kg} \mathrm{ha}^{-1} \mathrm{NPKF}$ & $6.53 \mathrm{~b}$ & $4.71 \mathrm{~b}$ & $0.84 \mathrm{~d}$ & $22.61 \mathrm{f}$ \\
$4 \mathrm{t} \mathrm{ha}^{-1} \mathrm{CPW}+200 \mathrm{~kg} \mathrm{ha}^{-1} \mathrm{NPKF}$ & $6.52 \mathrm{c}$ & $4.39 \mathrm{~d}$ & $0.83 \mathrm{e}$ & $23.12 \mathrm{c}$ \\
$4 \mathrm{t} \mathrm{ha}^{-1} \mathrm{CPW}+100 \mathrm{~kg} \mathrm{ha}^{-1} \mathrm{NPKF}$ & $6.34 \mathrm{e}$ & $4.38 \mathrm{e}$ & $0.76 \mathrm{f}$ & $21.65 \mathrm{~g}$ \\
$4 \mathrm{t} \mathrm{ha}^{-1} \mathrm{KPH}+200 \mathrm{~kg} \mathrm{ha}^{-1} \mathrm{NPKF}$ & $6.06 \mathrm{~g}$ & $4.34 \mathrm{f}$ & $0.68 \mathrm{i}$ & $22.81 \mathrm{e}$ \\
$4 \mathrm{t} \mathrm{ha}^{-1} \mathrm{KPH}+100 \mathrm{~kg} \mathrm{ha}^{-1} \mathrm{NPKF}$ & $6.37 \mathrm{~d}$ & $4.34 \mathrm{f}$ & $0.76 \mathrm{f}$ & $22.86 \mathrm{~d}$ \\
$2 \mathrm{t} \mathrm{ha}^{-1} \mathrm{WM}+200 \mathrm{~kg} \mathrm{ha}^{-1} \mathrm{NPKF}$ & $6.03 \mathrm{~h}$ & $3.21 \mathrm{~g}$ & $0.61 \mathrm{j}$ & $19.85 \mathrm{i}$ \\
$2 \mathrm{t} \mathrm{ha}^{-1} \mathrm{WM}+100 \mathrm{~kg} \mathrm{ha}^{-1} \mathrm{NPKF}$ & $6.04 \mathrm{i}$ & $2.97 \mathrm{~h}$ & $0.62 \mathrm{k}$ & $17.63 \mathrm{j}$
\end{tabular}

Note: $\mathrm{NPKF}=$ NPK 15:15:15 fertilizer; $\mathrm{CBH}=$ Cocoa Bean Husk; $\mathrm{CPH}=$ Cocoa Pod Husk; CPW= Cocoa Pod Waste; $\mathrm{KPH}=$ Kola Pod Husk; WM= Weed Mulch. Means with the same letters are not significantly different $(P<0.05)$ from other.

Table 6. Effects of combined amendments on soil K, $\mathrm{Ca}, \mathrm{Mg}$ and $\mathrm{Na}$ at Ile-Oluji.

\begin{tabular}{|c|c|c|c|c|}
\hline Treatments & $\left.\mathbf{K}(\mathbf{c m o l ~ k g})^{-1}\right)$ & $\mathrm{Ca}\left(\mathrm{cmol} \mathrm{kg}^{-1}\right)$ & $\mathrm{Mg}\left(\mathrm{cmol} \mathrm{kg}^{-1}\right)$ & $\mathrm{Na}\left(\mathrm{cmol} \mathrm{kg}^{-1}\right)$ \\
\hline Control & 0.411 & $1.42 \mathrm{j}$ & 0.371 & $0.69 \mathrm{e}$ \\
\hline $300 \mathrm{~kg} \mathrm{ha}^{-1} \mathrm{NPKF}$ & $0.86 \mathrm{~d}$ & $1.68 \mathrm{~b}$ & $0.46 \mathrm{k}$ & $0.67 \mathrm{~g}$ \\
\hline $4 \mathrm{t} \mathrm{ha}^{-1} \mathrm{CBH}+200 \mathrm{~kg} \mathrm{ha}^{-1} \mathrm{NPKF}$ & $0.88 \mathrm{c}$ & $1.64 \mathrm{e}$ & $0.79 \mathrm{a}$ & $0.75 \mathrm{a}$ \\
\hline $4 \mathrm{t} \mathrm{ha}^{-1} \mathrm{CBH}+100 \mathrm{~kg} \mathrm{ha}^{-1} \mathrm{NPKF}$ & $0.90 \mathrm{~b}$ & $1.45 \mathrm{i}$ & $0.75 \mathrm{~d}$ & $0.72 \mathrm{c}$ \\
\hline $4 \mathrm{t} \mathrm{ha}^{-1} \mathrm{CPH}+200 \mathrm{~kg} \mathrm{ha}^{-1} \mathrm{NPKF}$ & $0.91 \mathrm{a}$ & $1.53 \mathrm{~h}$ & $0.79 \mathrm{a}$ & $0.68 \mathrm{f}$ \\
\hline $4 \mathrm{t} \mathrm{ha}^{-1} \mathrm{CPH}+100 \mathrm{~kg} \mathrm{ha}^{-1} \mathrm{NPKF}$ & $0.83 \mathrm{e}$ & $1.45 \mathrm{a}$ & $0.77 \mathrm{c}$ & $0.73 b$ \\
\hline $4 \mathrm{t} \mathrm{ha}^{-1} \mathrm{CPW}+200 \mathrm{~kg} \mathrm{ha}^{-1} \mathrm{NPKF}$ & $0.72 \mathrm{~h}$ & $1.67 \mathrm{~d}$ & $0.74 \mathrm{e}$ & $0.68 \mathrm{f}$ \\
\hline $4 \mathrm{t} \mathrm{ha}^{-1} \mathrm{CPW}+100 \mathrm{~kg} \mathrm{ha}^{-1} \mathrm{NPKF}$ & 0.691 & $1.64 \mathrm{e}$ & $0.73 \mathrm{i}$ & $0.69 \mathrm{e}$ \\
\hline $4 \mathrm{t} \mathrm{ha}^{-1} \mathrm{KPH}+200 \mathrm{~kg} \mathrm{ha}^{-1} \mathrm{NPKF}$ & $0.76 \mathrm{f}$ & $1.73 \mathrm{a}$ & $0.77 \mathrm{c}$ & $0.70 \mathrm{~d}$ \\
\hline $4 \mathrm{t} \mathrm{ha}^{-1} \mathrm{KPH}+100 \mathrm{~kg} \mathrm{ha}^{-1} \mathrm{NPKF}$ & $0.65 \mathrm{j}$ & $1.69 \mathrm{c}$ & $0.71 \mathrm{i}$ & $0.68 \mathrm{f}$ \\
\hline $2 \mathrm{t} \mathrm{ha}^{-1} \mathrm{WM}+200 \mathrm{~kg} \mathrm{ha}^{-1} \mathrm{NPKF}$ & $0.75 \mathrm{~g}$ & $1.62 \mathrm{f}$ & $0.78 b$ & $0.64 \mathrm{~h}$ \\
\hline $2 \mathrm{t} \mathrm{ha}^{-1} \mathrm{WM}+100 \mathrm{~kg} \mathrm{ha}^{-1} \mathrm{NPKF}$ & $0.56 \mathrm{k}$ & $1.54 \mathrm{~g}$ & $0.69 \mathrm{j}$ & $0.63 \mathrm{i}$ \\
\hline
\end{tabular}

Note: NPKF= NPK 15:15:15 fertilizer; $\mathrm{CBH}=$ Cocoa Bean Husk; CPH= Cocoa Pod Husk; CPW= Cocoa Pod Waste; $\mathrm{KPH}=$ Kola Pod Husk; WM= Weed Mulch. Means with the same letters are not significantly different $(P<0.05)$ from other.

The result on the effect of soil amendments on soil OM revealed that the highest value of OM was recorded for the plot amended with $4 \mathrm{tha}^{-1} \mathrm{CBH}+200$ or $100 \mathrm{~kg} \mathrm{ha}^{-1} \mathrm{NPKF}$ and it showed a significant difference compared with plot treated with other soil amendments. The least value of soil organic matter was recorded for the control and it was significantly lower than other soil amendments at $(\mathrm{P}<0.05)$ (tables 5 and 6$)$. Values on soil $\mathrm{N}$ revealed that $(0.93,0.90$ and $0.89 \%) \mathrm{N}$ were obtained for $4 \mathrm{t} \mathrm{ha}^{-1} \mathrm{CPH}$ and CBH plus $200 \mathrm{~kg} \mathrm{ha}^{-1} \mathrm{NPKF}$ and $300 \mathrm{~kg} \mathrm{ha}^{-1} \mathrm{NPKF}$ respectively and they were significantly different from one another and other amendments imposed. The least value of $\mathrm{N}$ was recorded for the control. The highest value of soil $\mathrm{p}$ was obtained for the plot treated with $300 \mathrm{~kg} \mathrm{ha}^{-1} \mathrm{NPKF}$ and it was significantly different from the $\mathrm{P}$ values recorded for other soil amendments used during the experiment. The control also recorded the least value of $\mathrm{P}$ which was lower significantly compared with other soil amendments. The result of soil exchangeable properties $(\mathrm{K}, \mathrm{Ca}, \mathrm{Mg}, \mathrm{Na}, \mathrm{H}$ and $\mathrm{Al}$ ) revealed that the highest value of soil $\mathrm{K}$ was recorded for $4 \mathrm{tha}$ ${ }^{1} \mathrm{CPH}+200 \mathrm{~kg} \mathrm{ha}^{-1} \mathrm{NPKF}$ and it showed a significant difference when compared with other soil amendments tested. The value of soil Ca was significantly different compared with other soil amendments. Soil $\mathrm{pH}, \mathrm{OM}, \mathrm{N}$ and $\mathrm{P}$ as affected by amendments are presented in table 4 . All the amendments tested significantly $(\mathrm{p}<0.05)$ increased soil $\mathrm{pH}$, $\mathrm{OM}, \mathrm{N}$ and $\mathrm{P}$ concentration relative to control after harvest. The concentration of soil $\mathrm{pH}, \mathrm{OM}, \mathrm{N}$ and $\mathrm{P}$ were significantly increased by $4 \mathrm{t} \mathrm{ha}^{-1} \mathrm{CBH}+200 \mathrm{~kg} \mathrm{ha}^{-1} \mathrm{NPKF}$ relative to other amendments while the least values of soil $\mathrm{pH}, \mathrm{OM}, \mathrm{N}$ and $\mathrm{P}\left(6.09,3.45,0.68\right.$ and 18.29) were respectively recorded for $2 \mathrm{t} \mathrm{ha}^{-1} \mathrm{WM}+100 \mathrm{~kg} \mathrm{ha}^{-1} \mathrm{NPKF}$.

Effects of amendments on soil exchangeable properties are presented in tables 6 and 7. All the amendments tested significantly improved soil $\mathrm{K}, \mathrm{Ca}, \mathrm{Mg}, \mathrm{Na}$, ECEC and BS relative to control after harvest. Among the fertilizer treatments, the highest $\mathrm{K}, \mathrm{Ca}, \mathrm{Mg}, \mathrm{Na}, \mathrm{ECEC}$ and $\mathrm{BS}(0.97,1.76,0.88,0.80,6.70$ and 65.82$)$ were respectively 
obtained for soil treated with $4 \mathrm{t} \mathrm{ha}^{-1} \mathrm{CBH}+200 \mathrm{~kg} \mathrm{ha}^{-1}$ NPKF and were significantly $(\mathrm{p}<0.05)$ higher than the values recorded for other amendments.

Table 7. Effects of combined amendments on soil H+, Al, ECEC and BS at Ile-Oluji.

\begin{tabular}{lllll}
\hline Treatments & $\mathbf{H}+\left(\mathbf{c m o l ~ k g}^{-1}\right)$ & $\mathbf{A l}\left(\mathbf{c m o l ~ k g} \mathbf{~ g}^{-1}\right)$ & $\mathbf{E C E C ~}\left({\left.\mathbf{c m o l ~} \mathbf{~ k g}^{-1}\right)}^{\mathbf{B S}(\%)}\right.$ \\
\hline Control & $1.97 \mathrm{a}$ & $0.75 \mathrm{a}$ & $5.52 \mathrm{j}$ & 50.441 \\
$300 \mathrm{~kg} \mathrm{ha}^{-1} \mathrm{NPKF}$ & $1.82 \mathrm{~b}$ & $0.71 \mathrm{~b}$ & $6.20 \mathrm{~b}$ & $59.19 \mathrm{k}$ \\
$4 \mathrm{t} \mathrm{ha}^{-1} \mathrm{CBH}+200 \mathrm{~kg} \mathrm{ha}^{-1} \mathrm{NPKF}$ & $1.77 \mathrm{c}$ & $0.46 \mathrm{~d}$ & $6.29 \mathrm{a}$ & $64.55 \mathrm{c}$ \\
$4 \mathrm{t} \mathrm{ha}^{-1} \mathrm{CBH}+100 \mathrm{~kg} \mathrm{ha}^{-1} \mathrm{NPKF}$ & $1.72 \mathrm{f}$ & $0.44 \mathrm{e}$ & $5.98 \mathrm{e}$ & $63.88 \mathrm{~g}$ \\
$4 \mathrm{t} \mathrm{ha}^{-1} \mathrm{CPH}+200 \mathrm{~kg} \mathrm{ha}^{-1} \mathrm{NPKF}$ & $1.74 \mathrm{~d}$ & $0.46 \mathrm{~d}$ & $6.10 \mathrm{c}$ & $63.77 \mathrm{~h}$ \\
$4 \mathrm{t} \mathrm{ha}^{-1} \mathrm{CPH}+100 \mathrm{~kg} \mathrm{ha}^{-1} \mathrm{NPKF}$ & $1.72 \mathrm{f}$ & $0.46 \mathrm{~d}$ & $5.96 \mathrm{f}$ & $63.42 \mathrm{i}$ \\
$4 \mathrm{t} \mathrm{ha}^{-1} \mathrm{CPW}+200 \mathrm{~kg} \mathrm{ha}^{-1} \mathrm{NPKF}$ & $1.73 \mathrm{e}$ & $0.46 \mathrm{~d}$ & $6.00 \mathrm{~d}$ & $63.33 \mathrm{j}$ \\
$4 \mathrm{t} \mathrm{ha}^{-1} \mathrm{CPW}+100 \mathrm{~kg} \mathrm{ha}^{-1} \mathrm{NPKF}$ & $1.66 \mathrm{~h}$ & $0.41 \mathrm{~g}$ & $5.82 \mathrm{~h}$ & $64.43 \mathrm{~d}$ \\
$4 \mathrm{t} \mathrm{ha}^{-1} \mathrm{KPH}+200 \mathrm{~kg} \mathrm{ha}^{-1} \mathrm{NPKF}$ & $1.67 \mathrm{~g}$ & $0.47 \mathrm{c}$ & $6.10 \mathrm{c}$ & $64.92 \mathrm{a}$ \\
$4 \mathrm{t} \mathrm{ha}^{-1} \mathrm{KPH}+100 \mathrm{~kg} \mathrm{ha}^{-1} \mathrm{NPKF}$ & $1.64 \mathrm{i}$ & $0.44 \mathrm{e}$ & $5.81 \mathrm{i}$ & $64.20 \mathrm{e}$ \\
$2 \mathrm{t} \mathrm{ha}^{-1} \mathrm{WM}+200 \mathrm{~kg} \mathrm{ha}^{-1} \mathrm{NPKF}$ & $1.62 \mathrm{j}$ & $0.44 \mathrm{e}$ & $5.83 \mathrm{~g}$ & $64.67 \mathrm{~b}$ \\
$2 \mathrm{t} \mathrm{ha}^{-1} \mathrm{WM}+100 \mathrm{~kg} \mathrm{ha}^{-1} \mathrm{NPKF}$ & $1.51 \mathrm{k}$ & $0.42 \mathrm{f}$ & $5.35 \mathrm{k}$ & $63.93 \mathrm{f}$ \\
\hline
\end{tabular}

Note: NPKF= NPK 15:15:15 fertilizer; $\mathrm{CBH}=$ Cocoa Bean Husk; CPH= Cocoa Pod Husk; CPW= Cocoa Pod Waste; $\mathrm{KPH}=$ Kola Pod Husk; WM= Weed Mulch. Means with the same letters are not significantly different $(P<0.05)$ from other.

\section{DISCUSSION}

Optimum yield could not be obtained from the sole application of organic manures due to the slow release of nutrients. The use of organic and inorganic fertilizers has been advocated for sustainable soil productivity under intensive and continuous cultivation in southwest Nigeria (Adepetu et al., 1997), sustainable nutrient management on smallholder farms can be achieved by a combination of mineral fertilizers and organic soil amendments (Vanauwe $e t$ al., 2004). From this study, the contribution of amendments to soil fertility has clearly shown through increased availability of plant nutrients for its growth and development (Brady \& Weil, 2007; Adeoluwa et al., 2010). A combination of organic and inorganic sources of nutrients led to optimal $\mathrm{pH}$ of about 6.0-7.0 which is recommended for nutrient availability and suitable for most tropical field and arable crops. It could be seen that a combination of $4 \mathrm{t} \mathrm{ha}{ }^{-1}$ $\mathrm{CBH}+200 \mathrm{~kg} \mathrm{ha}^{-1} \mathrm{NPKF}, 4 \mathrm{t} \mathrm{ha}^{-1} \mathrm{CBH}+100 \mathrm{~kg} \mathrm{ha}^{-1} \mathrm{NPKF}$ and $4 \mathrm{t} \mathrm{ha}^{-1} \mathrm{CBH}+200 \mathrm{~kg} \mathrm{ha} \mathrm{NPKF}^{-1} \mathrm{gave}$ highest value of soil N, P and $\mathrm{K}$ which are the nutrients most often limiting to tropical crops and deficient tropical soils (especially $\mathrm{N}$ and P). The improved soil chemical properties should have enhanced nutrients uptake by melon. The better attributes of the combination of nutrient sources are further confirmed by the relatively high soil organic matter contents and in the cases of $4 \mathrm{t} \mathrm{ha}^{-1} \mathrm{CBH}$ plus any of 200 or $100 \mathrm{~kg} \mathrm{ha}^{-1}$ NPKF. The integrated soil fertility management (ISFM) approach supports that there are positive interrelations and complementarities of organic and mineral nutrient sources to support sustained soil fertility and crop productivity. The trend in the values of soil chemical properties indicated that higher values of soil organic matter were more favourable to soil quality hence melon performance. The result of the experiment also showed that soil amendments improved soil fertility and increased growth components of white seed melon relative to control. This is consistent with the findings of Liu \& Stutzel (2004) and Adeoluwa et al. (2010). The result is also in agreement with that of Prasad \& Singh (2000) who reported that soils treated with soil amendments increased number of leaves and other growth components compared with untreated soil.

\section{CONCLUSION}

Combination of selected plant residues with synthetic fertilizer (NPK 15:15:15) showed improving effects on soil chemical properties and growth components of white seed melon compared with the control. Combination of $4 \mathrm{t} \mathrm{ha}^{-1}$ $\mathrm{CBH}+200 \mathrm{~kg} \mathrm{ha}^{-1} \mathrm{NPKF}, 4 \mathrm{t} \mathrm{ha}^{-1} \mathrm{CPW}+200 \mathrm{~kg} \mathrm{ha}^{-1} \mathrm{NPKF}$ and $4 \mathrm{t} \mathrm{ha}^{-1} \mathrm{CPH}+200 \mathrm{~kg} \mathrm{ha}^{-1} \mathrm{NPKF}$ gave highest value of soil N, P, K, Ca, Mg, ECEC and BS respectively compared to others. The overall results revealed that a combination of plant residues with NPKF particularly $4 \mathrm{t} \mathrm{ha}^{-1} \mathrm{CBH}+200 \mathrm{~kg} \mathrm{ha}^{-1} \mathrm{NPKF}$ can be used for improving soil with profitable production of white seed melon in the study area.

\section{ACKNOWLEDGEMENTS}

The Authors are grateful for the support received from the Teaching and Research Farm, Federal Polytechnic Ile Oluji, Ondo State.

\section{REFERENCES}

Adekiya A.O., Ojeniyi S.O. \& Agbede M.T. (2012). Poultry manure effects on soil properties, leaf nutrient status, growth and yield of cocoyam in a tropical Alfisol. Nigerian Journal of Soil Science, 22(2): 30-39. 
Adeoluwa O.O., Adeoye G.O. \& Yusuf S.A. (2010). Effects of organic nitrogen fortified on some growth parameters of green Amaranthus. Journal of Renewable Agriculture and food Systems, 24(4): 245-250.

Adepetu J.A., Aduayi E.A. \& Alofe C.O. (1997). Preliminary survey of the fertility status of soil in Ondo state under traditional cultivation. Ife Journal of Agriculture, 1: 134-149.

Agbede O.O. \& Kalu B.A. (1995). Constrains of small scale farmers in increasing crop yields, farm size and fertilizer supply. Nigeria Journal of Soil Science, 11:139-152.

Akanbi O.S., Famaye A.O., Ojeniyi S.O., Nduka B.A., Taiwo N., Olasupo F.O., Azeez O.M., Edibo G.O. \& Gbemisola D.O. (2013). Growth response of Cashew seedlings to applied organic fertilizer of animal and plant origin on degraded soil in Ibadan, Southwest Nigeria. International Research Journal of Agricultural Science and Soil Science, 3(3): 93-98.

Akinrinde E.A. \& Obigbesan G.O. (2000). Evaluation of the fertility status of selected soils for crop production in five ecological zones of Nigeria. In: Proceedings of the $26^{\text {th }}$ Annual Conference of Soil Science Society of Nigeria, Ibadan, Nigeria, pp. 279-288.

Babadele D.O. \& Ojeniyi S.O. (2013). Cassava yield as effects by sawdust ash and siamweed residue and NPK fertilizer. Nigerin Journal of Soil Science, 23(2): 282-290.

Boyd E. (1996). Soil Fertility, Second Edition by Publisher: CRC Press.

Brady N.C. \& Weil R.R. (2007). The Nature and Properties of soils. Prentice hall, Inc., upper saddle River, NJ, pp. 18-23.

Bremner J.M. (1996). Nitrogen-total. In: Sparks D.L. (Ed.) Methods of Soil Analysis. Part 3. Chemical Methods. $2^{\text {nd }}$ Ed. SSSA Book Series No. 5, ASA and SSSA, Madison, WI, USA. pp. 1085-1121.

Carter M.R. (1993). Soil sampling and methods of analysis. Canadian Society of Soil Science, Lewis Publishers London, pp. 823.

Cezar P.M. (2004). Organic Fertilizer in Rice: Myths and facts. A public education series of the Asia Rice foundation.

Ellison J (2011). http://www.basic-info-4-organic-fertilizers.com/

Frank K., Beegle D. \& Denning J. (1998). Phosphorus. In: Brown J.R. (Ed.) Recommended Chemical Soil Test Procedures for the North Central Region, North Central Regional Research Publication No. 221 (revised). Missouri Agric. Exp. Stn., Columbia, MO, pp. 21-26.

Hendershot W.H. \& Lalande H. (1993). Ion exchange and exchangeable cations. In: Carter M.R. (Ed.) Soil Sampling and Methods of Analysis. Canadian Society of Soil Science, Lewi Publishers, London, pp. 167-176.

Liu F. \& Stutzel H. (2004). Biomass partitioning, Specific leaf area and water use efficiency of vegetable amaranth (Amaranthus spp.) in response to drought stress. Science and Horticulture, 102: 15-27.

National Research Council (2006). Lost Crops of Africa: Volume II: Vegetables. Washington, DC: The National Academies Press. Retrieved from: https://doi.org/10.17226/11763.

Nelson D.W. \& Sommers L.E. (1996). Total carbon, organic carbon and organic matter. In: Sparks D.L. (Ed.) Methods of Soil Analysis. Part 3, $2^{\text {nd }}$ Ed. SSSA Book Series No. 5, SSSA, Madison, WI., USA, pp. 961-1010.

Ojeniyi S.O. \& Adeniyan N.O. (1999). Effect of poultry manure and NPK fertilizer on soil fertility, nutrient contents and yield of maize at Akure South West Nigeria in: Management of the soil resources of Nigeria for sustainable agricultural production in the $21^{\text {st }}$ century. In: Proceedings of the $25^{\text {th }}$ Annual Conference of the Soil Science Society of Nigeria (SSSN), Benin City, Nigeria, pp. 185-191.

Ojeniyi S.O. (2000). Effect of goat manure on soil nutrients and okra yield in a rainforest area of Nigeria. Applied Tropical Agriculture, 5: 20-23.

Okigbo I. (2000). Application of organic and inorganic fertilizers and the response of maize crop. Nigerian Journal of Soil Science, 18(2): 22.

Onunka N.A. \& Nwokocha H.N. (2003). varietal response of sweet potato (Ipomoea batat (L) Lam) to different levels of nitrogen fertilization in tropical ultisol of south Eastern Nigeria. Journal of Science and Technology Research, (2)2: 3003.

Prasad B. \& Singh S.K. (2000). Long term effects of fertilizers and organic manures on crop yields, nutrients balance and soil properties in rice - wheat cropping system in Bihar. In: long - term soil fertility Experiment in Rice - Wheat cropping system. Advances in Soil Science, 20: 105-119.

Sheldrick B.H. and Wang C. (1993). Particle-size distribution. In: Carter M.R. (Ed.) Soil Sampling and Methods of Analysis. Canadian Society of Soil Science, Lewis Publishers, Ann Arbor MI, pp. 495-511.

Vanauwe B., Diels J., Sanginga N. \& Merckx R. (2004). Integrated Plant Nutrient Management in Sub-saharan Africa: From Concept to Practice. CABI Publishing. Oxon, U.K., pp. 352. 